\title{
Conventional kinesiotherapy or Pilates mat exercises, what is more efficient for abdominal weakness? A feasibility study
}

\author{
Renan Lima Monteiro ${ }^{1}$, Laio Braga de Oliveira ${ }^{2}$, Luiz Armando Vidal Ramos ${ }^{1 *}$, \\ Mauricio Oliveira Magalhães ${ }^{1}$, Marília Maniglia de Resende ${ }^{2}$, Bianca Callegari ${ }^{3}$ \\ ${ }^{1}$ Department of Physical, Speech and Occupational Therapy, School of Medicine of University of Sao Paulo, Sao Paulo, Brazil \\ ${ }^{2}$ Educational Association of Amazonia-SEAMA, Amapá, Brazil \\ ${ }^{3}$ Department of Physical Therapy and Occupational Therapy, Institute of Health Sciences, University Federal of Pará, Belém, Brazil \\ Email: ${ }^{*}$ luiz.armando@usp.br
}

Received 29 October 2013; revised 28 November 2013; accepted 9 December 2013

Copyright (C) 2013 Renan Lima Monteiro et al. This is an open access article distributed under the Creative Commons Attribution License, which permits unrestricted use, distribution, and reproduction in any medium, provided the original work is properly cited. In accordance of the Creative Commons Attribution License all Copyrights (C) 2013 are reserved for SCIRP and the owner of the intellectual property Renan Lima Monteiro et al. All Copyright (C 2013 are guarded by law and by SCIRP as a guardian.

\begin{abstract}
The aim of this feasibility study was to contrast rectus abdominis (RA) muscle strength and electrical activity after two abdominal training protocols, conventional kinesiotherapy (CK) and Pilates mat exercises (PME). 13 participants were randomized to one of two groups: CK and PME, and were trained accordingly. The upper rectus abdominis (URA) and lower rectus abdominis (LRA) were independently examined and inter and intra group comparisons were done. Findings demonstrate a significant increase in RA strength after both protocols, relative to baseline. Significantly increased electrical activation was seen in the URA after CK. Training with PME, although as efficient as $\mathrm{CK}$ in strength improvement, produced decreased activation level of muscles. CK training induced an opposite result. The differences after training suggest that $\mathrm{CK}$ and PME training programmers may strength RA, but the neuromuscular activation strategies for that are not the same. PME can be incorporated into protocols to improve abdominals strength, and trunk stabilization.
\end{abstract}

Keywords: Rectus Abdominis; Pilates Mat Exercise; Surface Electromyography

\section{INTRODUCTION}

Pilates mat exercise (PME) has become a fast-growing popular trend in rehabilitation and conditioning programs.

${ }^{*}$ Corresponding author.
It aims to improve flexibility, increase global muscular strength and endurance, by emphasizing spinal and pelvic alignment, proper breathing, and the development of a strong core or center (tones abdominals while strengthening the back). PME is considered to improve coordination and balance and is claimed to increase activation of abdominal muscles during athletics or daily living activities [1-3]. Joseph Pilates (1880-1967) developed this method seeking to match scientific principles of physical training with oriental philosophies and movement techniques $[4,5]$.

The rectus abdominis muscle (RA) is a superficial and important postural muscle, which also stabilizes pelvic movements, assists in breathing and supports maintenance of proper intra-abdominal pressure. When abdominal strength is not adequate to counter the pull of the antagonist Erector Spine under load, these low back muscles are put at a mechanical disadvantage further placing additional stresses on lower back $[6,7]$.

Conventional kinesiotherapy (CK) is often used for strengthening the RA, since this seems to be associated with decreased risk of lumbar spine lesions, or with improved rehabilitation after these lesions [8-11].

Both CK and PME focus on several deep and superficial muscles, including the RA. A better comprehension of how the different types of exercises recruit the RA is a necessary step for the development of specific abdominal routines that better attend customized needs of patients or trainees [2,11-13]. Several studies investigated the effectiveness of $\mathrm{CK}$ in activating the RA for strength, pain relief, and rehabilitation after specific lesions [6-8, $10,14]$. Few studies focused, however, on the effective- 
ness of PME in improving abdominis muscular activity. Preview findings suggested low back pain, muscular strength and dynamic balance improved following Pilates, but rectus abdominis muscle activity was never measured, especially with sEMG [1-4,15-19]. Critchley et al. proposed, in 2011, the first prospective study into the effects of PME on abdominal muscles. Using ultrasound to measure Transverses abdominal (TrA) and Oblique internal abdominal thickness, they found that PME training appears to increase TrA activity during the exercises but not during functional postures. There was no mention, however, about the muscles' electrical activity patterns [20].

The aim of this feasibility study was to compare the effects of PME and a conventional strength training program of CK on the RA electrical activity and force. Scientific evidence to support the differences between these methods is yet missing. The secondary objective of this study was to contrast muscular activity in the upper (URA) and lower portions (LRA) of the RA after each intervention.

\section{METHODS}

\subsection{Experimental Approach to the Problem}

This study attempted to identify differences in force development and also in muscle recruitment patterns among two abdominal exercise methods. The Pilates method has been gaining recognition recently as a novel choice for strength and/or endurance of abdominal and lumbar muscles $[2,3]$. Few studies have investigated the electromyographic activity and strength during PME, and none of them had this follow-up investigation [18,21]. CK, however, includes several modes of exercises to improve strength an has been well investigated $[9,10,22$, 23]. By comparing both methods in a longitudinal study, differences in the electrical activation associated to the force generated may be identified. The results of this study are relevant to head coaches, strength coaches, coaches, athletic trainers and physical therapists in the fact that they may use different kinds of exercises concerning their aims. See Figure 1.

\subsection{Participants}

This feasibility study included participants were randomized to one of two groups: conventional kinesiotherapy (CK) and Pilates mat exercises (PME). Abdominal hypotonic was estimated using the Canadian Standardized Test of Fitness [24]. Participants were asked to conduct a series of abdominal flexions and were included if incapable of doing at least 24 repetitions in one minute. This study was conducted in the Clinical Assessment Laboratory of "Faculdade Seama", and participants agreed to participate by signing informed consent. The study

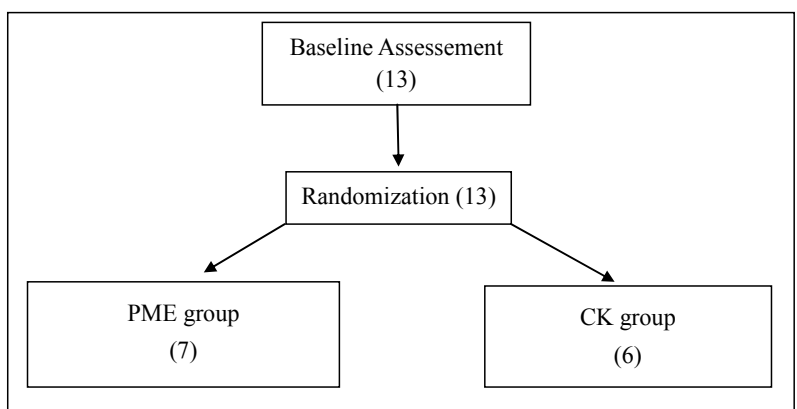

Figure 1. Study design flow chart.

was conducted in accordance to the Health National Council guidelines (Resolution 196/96).

\subsection{Procedures}

Firstly, all basic anthropometric measurements were collected and the subjects were familiarizes with the tasks and methods. The same investigator performed all procedures and tasks with each subject to ensure uniformity. All exercises were explained and demonstrated by an instructor trained in Pilates method by Metacorupus Studio Pilates ${ }^{\circledR}$.

Maximal isometric voluntary contraction (MIVC) was used to measure the RA force and URA and LRA electrical activity at baseline. The test was performed 3 times and the average of electrical activity and force parameters was used for further comparisons.

MIVC was conducted with participants in dorsal decubitus, with hip and knees straight and strapped with a belt. The measurement position was resisted curl-up with maximal isometric resistance applied to the shoulders [25]. Arms were crossed over the chest. RA maximal force contraction was measured during the test by digital dynamometer (Berman load cells, Primax Inc. São Paulo-SP) with maximal capacity of $200 \mathrm{Kgf}$, attached bellow the bed. Surface electromyography (sEMG) was used to measure muscle activity.

\section{4. sEMG Measurements}

An 8-resolution channel data acquisition system (model EMG820C, Emgsystem Inc; São José dos Campos, Brazil), consisting of a signal conditioner with a band-pass filter of $20-450 \mathrm{~Hz}$, and amplifier gain of 2000, was used to obtain the biological signals. All data were processed for analysis by specific software (Labview Emgsystem, Inc). sEMG activity was captured by differential surface electrode (SDS500), and performed by an A/D board with 14-bit resolution input range, sampling frequency of $2000 \mathrm{~Hz}$, common rejection module greater than $100 \mathrm{~dB}$, signal noise less than $0.3 \mu \mathrm{V}$ RMS and impedance of $109 \Omega$.

The electrodes $(20 \mathrm{~mm}$ diameter and centre-to-centre 
distance $20 \mathrm{~mm}$ ) were placed bilaterally in the ventral portion of the upper and lower parts muscle, and verticalized according to the guidance of the Surface EMG for the Non-invasive Assessment of Muscles. The skin was shaved, sanded, and swabbed with alcohol-soaked cotton before electrode placement to minimize skin resistance. The reference electrode was applied to the lateral malleolus of the dominant leg [26].

All data processing was performed and analyzed using MATLAB routines (Matlab10; The Mathworks Inc., Natick, MA). The EMG signal was processed to the root mean square (RMS) using a moving window of 50 milliseconds. For each portion, URA and LRA, the mean RMS value across all MVIC trials represented the maximal activity $\left(\mathrm{RMS}_{\mathrm{MVIC}}\right)$. $\mathrm{RMS}_{\mathrm{MVIC}}$ values were normalized by maximum peak (RMS MVICPEAK ) obtained during contraction of each portion of muscle using de following equation [27]:

$$
\text { Activation level }(\%)=\left[\mathrm{RMS}_{\mathrm{MVIC}} / \mathrm{RMS}_{\text {MVICPEAK }} \times 100\right]
$$

The EMG data were collected bilaterally from the URA and LRA. The mean of the two values was obtained and used for comparison between PME and CK.

After the baseline assessments, women were randomized to one of the two groups. A total of 15 sessions (three times per week for 5 weeks) were conducted. Patients were then reassessed following the same protocol and measurements were contrasted against baseline and between groups.

\subsection{Pilates Mat Exercises and Conventional Kinesiotherapy}

Following randomization, participants received information about their exercise programs and were encouraged to progress the exercises for 5 weeks, without absences. Volunteers who missed any of the sessions, for any reason, were excluded from the final sample.

PME consisted of conducting three exercises (The Hundred, One Leg Stretch, and One Leg Circle) under individual supervision of the therapist. Progressions were permitted whereby load could be increased changing limb movements or position.

The Hundred focuses on strengthening the RA and the external oblique muscles. Patients were positioned in dorsal decubitus, in crook lying, arms by side. They were asked to draw-in abdominal muscles, raising heads and shoulders from the mat. The arms should be raised and pulsed up and down while breathing. Volunteers could progress to lifting legs to $90^{\circ}$, but keeping the lower back on the mat and the abdominals flat. Three series of 30 repetitions were conducted.

One Leg Stretch aimed to strength the RA, external oblique and rectus femoris muscle. Participants started in crook lying and arms by side. They were asked to draw- in abdominal muscles, lifting straighten alternate legs to full knee extension. Volunteers could progress to starting with both legs in $90^{\circ}$. Three series of 30 repetitions were conducted.

On leg circle aims to strength the RA, external oblique, rectus femoris, iliopsoas, sartorius, tensor fasciae latae, pectineus, anterior fibers of the gluteus medius, and gluteus minimus. Volunteers started in the same position from One Leg Stretch, but now they should describe 5 small circles with raised leg. Volunteers could progress to starting with both legs in $90^{\circ}$, and circle only one leg. Three series of 10 repetitions were conducted.

CK consisted of three abdominal exercises (flexion, modified flexion, and abdominal paddling exercises). Abdominal flexion aimed to strength the RA and flexors of the hip. Volunteers were positioned in dorsal decubitus with knees in flexion and hands on the back of the head. Abdominal flexion was conducted in order to bring the chest to the vertical position. Three series of 10 repetitions were conducted.

Modified abdominal flexion also aimed to strength the RA and flexors of the hip. Different than in traditional abdominal flexion, hands were over the chest, and elevation of the chest was up to $30^{\circ}$. Three series of 10 repetitions were conducted.

Abdominal paddling exercises focused on strengthening the RA. Arms were extended above the head and legs were in extension. Simultaneous flexion of the trunk and legs were conducted, and arms were extended until being parallel to the ground. Three series of 10 repetitions were conducted.

\subsection{Statistical Analyses}

Sample size was estimated using SigmaStat 3.5. Considering the mean and standard initial RMS founded and assuming a confidence interval of $95 \%$ and power of $80 \%$, required sample was determined as being 12 . The Shapiro-Wilk test was used to assess data distribution (normality test). The $\mathrm{t}$ Student test compared groups. Significance was determined at the 0.05 level.

\section{RESULTS}

This feasibility study included 13 participants where 7 participants in the Pilates mat exercises (PME) group and 6 in the conventional kinesiotherapy (CK) group. Table 1 displays the demographic data. Groups were similar regarding age, body mass index, body fat and baseline characteristics.

Figure 2 displays the pre-and post intervention data in those in the PME group. Although there was a decreasing tendency, activation level was not significantly different for the URA $(p=0.428)$ and LRA $(p=0.109)$. Strength of the RA increased significantly with training 
Table 1. Characteristics of the population.

\begin{tabular}{cccc}
\hline Variables & PME $(\mathbf{n}=\mathbf{7})$ & CK $(\mathbf{n}=\mathbf{6})$ & $\mathbf{p}$ \\
\hline Age (years) & $27.0 \pm 4.4$ & $25.0 \pm 3.1$ & 0.45 \\
BMI $\left(\mathrm{Kg} / \mathrm{m}^{2}\right)$ & $21.6 \pm 1.6$ & $21.7 \pm 1.9$ & 0.68 \\
Abdominal flexions & $18.0 \pm 4.8$ & $16.6 \pm 5.3$ & 0.80 \\
\% Body fat & $28.2 \pm 0.9$ & $27.6 \pm 3.3$ & 0.65 \\
\hline
\end{tabular}

$\mathrm{p}$-value for $\mathrm{t}$ test; Mean and standard deviation: age, BMI = body mass index, abdominal flexions and $\%$ body fat.

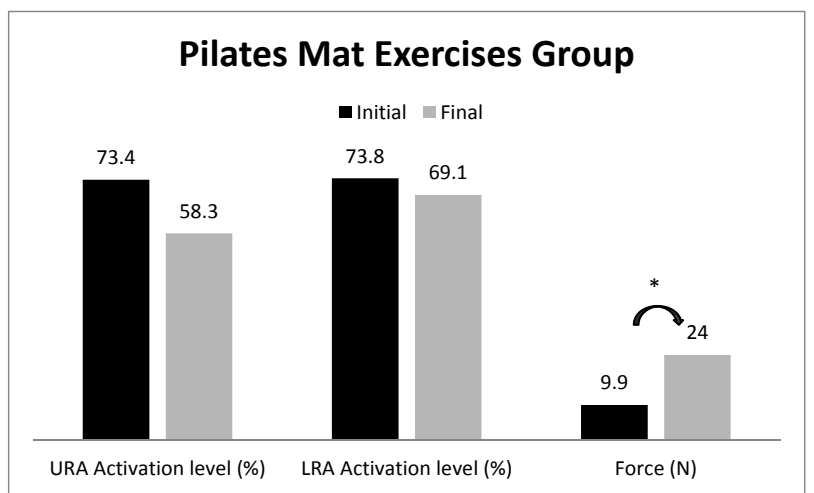

Figure 2. Pilates mat exercise group-activation level (\%) and force $(\mathrm{N})$ RA values (URA and LRA, significant differences marked $\mathrm{p}<0.05)$.

$(\mathrm{p}=0.004)$.

Figure 3 displays similar data in those receiving CK. Results were significant for URA $(p=0.042)$ but not for LRA ( $p=0.373)$. RA strength improved significantly with training $(p=0.001)$.

Contrasts between groups are displayed in Figure 4. No significant differences were seen for activation level of the LRA ( $p=0.431)$. URA, in the PME group, had significant decreased activation level compared to those in CK group, who had increased activation $(p=0.014)$. Strength improved in both groups after intervention and differences were not significant between groups $(\mathrm{p}=$ $0.885)$.

\section{DISCUSSION}

The aim of this feasibility study was to contrast rectus abdominis (RA) muscle strength and electrical activity after two abdominal training protocols, conventional kinesiotherapy (CK) and Pilates mat exercises (PME). The results demonstrated that both interventions were capable of strengthen the RA, and there were no significant difference in the amount of strength improved using PME or CK programmers. The characteristics of the electrical activity from the different portions of RA, however, suggested that there are distinct patterns of muscle recruitment for the production of this force. The differences after 5 weeks also suggests that distinct training programmers may strength RA, but the neuromuscu-

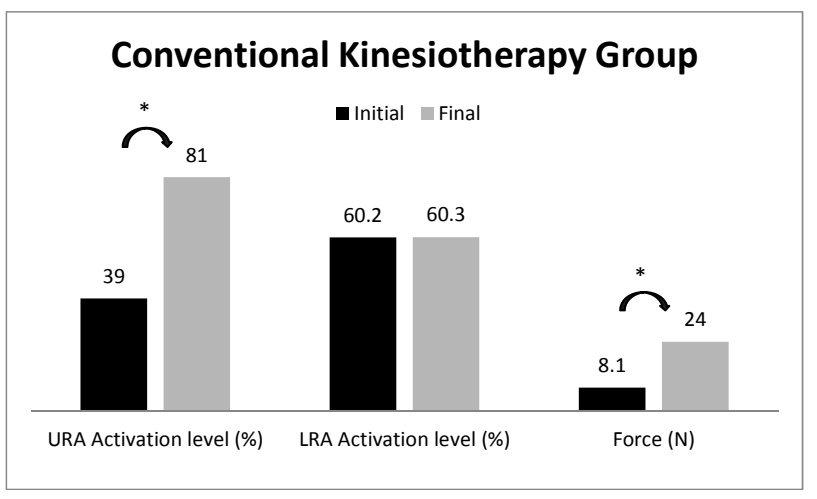

Figure 3. Conventional kinesiotherapy group-activation level (\%) and force $(\mathrm{N})$ RA values (URA and LRA, significant differences marked $\mathrm{p}<0.05)$.

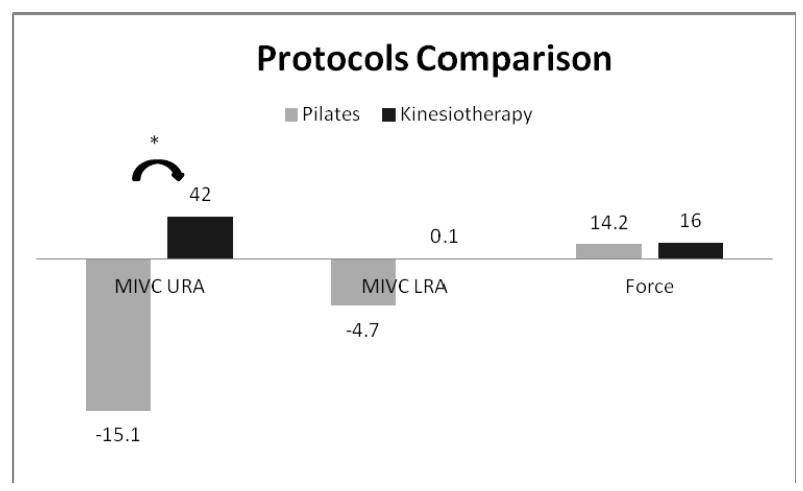

Figure 4. Pilates mat exercise and conventional kinesiotherapy groups comparison-final-initial activation level (\%) and finalinitial force $(\mathrm{N})$ RA values (MIVC URA and MIVC LRA, significant differences marked $p<0.05)$.

lar activation strategies for that are not the same. Training with PME, although as efficient as $\mathrm{CK}$ in strength improvement, produced decreased activation level of muscles after 5 weeks. CK training, on the other hand, induced an opposite result.

Since one of our goals was to contrast RA electrical activity post-interventions, relative to baseline, results suggest that only URA had significant improvement after CK, while LRA portion showed a non-significant trend. Abdominal muscular activation following $\mathrm{CK}$ is well demonstrated [8-10,14,23,25,28,29]. Findings suggest that superior chains of the RA are activated during abdominal flexion, demonstrating that this muscle is requested during exercise [28,30]. Axler and McGill [8] also found a differential pattern of activation for the superior and inferior parts of the RA during different types of abdominal exercises, and high levels of activity under specific exercise conditions (trunk curl), suggesting the benefit of these exercises relative to sit-up exercise types. Other studies, on the other hand, failed to demonstrate the difference [31].

The differential response of the URA and LRA to CK 
may be explained by the fact that $\mathrm{CK}$ was conducted under higher speed than PME, which emphasizes paced and synchronic rhythms [5,32]. Accordingly, CK probably recruited a higher number of motor units during exercises, relative to PME [22]. These results are in agreement with others [23], which suggested that pace of exercising and numbers of motor units are directly correlated. Comfort et al. [33] also suggest that maximal speed of RA contraction is associated with increased electrical activity, relative to slow contraction paradigms. Evidence also suggests the influence of force and speed in the EMG signal during dynamic exercises [34]. Accordingly, CK probably activated a higher number of fibers during training, reflecting in increased activation during MIVC.

Bloomer and Goldfarb [35] suggest that eccentric muscular action is associated to micro lesions. PME aims to strength agonists and antagonists trough eccentric muscular action $[2,16,18]$. It may be that those in the PME group were more likely to have these lesions and the electrical activity captured the regenerative response. Differently, those in the CK group had increased strength and this is supported by the fact that the superior portion of the RA is intensively activated by exercises of the trunk-curl type [10]. It has also been suggested that in order to increase strength, increased electrical activity and increased frequency of neuronal activation are simultaneously required [13]. Pandofe et al. [36] found the relationship between RA strength and RA electrical activities by demonstrating that women with level 5 of strength had EMG activity that was above normal. Therefore our findings are in alignment with the literature.

Most studies on abdominal exercises are focused on biomechanics and movements [22,23,28,33], and few focused on the effectiveness of specific training protocols, making it difficult to put our findings in context. Those in the PME group had significant increase in their strength, and this has been demonstrated by others $[3,10$, $20,37]$, in support to our findings. It is difficult to explain however, the de-linkage between strength and electrical activity decreased evidenced by our data. Few studies had focused in electrical activity of muscles with PME, and those who did, are cross-sectional studies that compared pattern of muscle recruitment during some trials of specific exercises $[21,27,38,39]$. This is the first longitudinal study evaluating activation level of RA in PME training. It may be that PME used other muscles in order to promote trunk flexion, including the internal and external oblique's, paravertebral chains, gluteus and quadriceps $[16,40]$. Altogether, they may have been synergistic to the movement and reduced overload on RA. In this regard we suggest that both CK and PME increase RA strength, albeit trough divergent principles.

\section{PRACTICAL APPLICATIONS}

The mat exercises require minimal equipment and can be accomplished in non-gym or clinical settings. Many Pilates exercises are similar to those employed in motor control and spinal stabilization training programmers. These findings suggest PME can be incorporated into protocols to improve abdominals strength force, and trunk stabilization. However the differences between the activation pattern recruitment should be further deep investigated.

\section{CONCLUSION}

CK and PME increased RA muscular strength. The inferior part of RA activation decreased following supervision PME, compared to the group following CK, where activation increased.

\section{REFERENCES}

[1] Blum, C.L. (2002) Chiropractic and Pilates therapy for the treatment of adult scoliosis. Journal of Manipulative and Physiological Therapeutics, 25, E3. http://dx.doi.org/10.1067/mmt.2002.123336

[2] Emery, K., De Serres, S.J., McMillan, A. and Côté, J.N. (2010) The effects of a Pilates training program on armtrunk posture and movement. Clinical Biomechanics (Bristol, Avon), 25, 124-130. http://dx.doi.org/10.1016/j.clinbiomech.2009.10.003

[3] Eyigor, S., Karapolat, H., Yesil, H., Uslu, R. and Durmaz, B. (2010) Effects of Pilates exercises on functional capacity, flexibility, fatigue, depression and quality of life in female breast cancer patients: A randomized controlled study. European Journal of Physical and Rehabilitation Medicine, 46, 481-487.

[4] (1999) Conditioning by Pilates. Harvard Women's Health Watch, 6, 7.

[5] Wells, C., Kolt, G.S. and Bialocerkowski, A. (2012) Defining Pilates exercise: A systematic review. Complementary Therapies in Medicine, 20, 253-262. http://dx.doi.org/10.1016/j.ctim.2012.02.005

[6] Nahhas Rodacki, C.L., Luiz Felix Rodacki, A., Ugrinowitsch, C., Zielinski, D. and Budal da Costa, R. (2008) Spinal unloading after abdominal exercises. Clinical Biomechanics (Bristol, Avon), 23, 8-14. http://dx.doi.org/10.1016/j.clinbiomech.2007.08.022

[7] Stokes, I.A., Gardner-Morse, M.G. and Henry, S.M. (2011) Abdominal muscle activation increases lumbar spinal stability: Analysis of contributions of different muscle groups. Clinical Biomechanics (Bristol, Avon), 26, 797803. http://dx.doi.org/10.1016/j.clinbiomech.2011.04.006

[8] Axler, C.T. and McGill, S.M. (1997) Low back loads over a variety of abdominal exercises: Searching for the safest abdominal challenge. Medicine and Science in Sports and Exercise, 29, 804-811.

http://dx.doi.org/10.1097/00005768-199706000-00011

[9] Escamilla, R.F., Babb, E., DeWitt, R., Jew, P., Kelleher, 
P., Burnham, T. and Imamura, R.T. (2006) Electromyographic analysis of traditional and nontraditional abdominal exercises: Implications for rehabilitation and training. Physical Therapy, 86, 656-671.

[10] Escamilla, R.F., McTaggart, M.S., Fricklas, E.J., DeWitt, R., Kelleher, P., Taylor, M.K. and Moorman, C.T. (2006) An electromyographic analysis of commercial and common abdominal exercises: Implications for rehabilitation and training. Journal of Orthopaedic \& Sports Physical Therapy, 36, 45-57. http://dx.doi.org/10.2519/jospt.2006.36.2.45

[11] Hides, J.A., Stanton, W.R., Wilson, S.J., Freke, M., McMahon, S. and Sims, K. (2010) Retraining motor control of abdominal muscles among elite cricketers with low back pain. Scandinavian Journal of Medicine \& Science in Sports, 20, 834-842.

http://dx.doi.org/10.1111/j.1600-0838.2009.01019.x

[12] Kloubec, J.A. (2010) Pilates for improvement of muscle endurance, flexibility, balance, and posture. Journal of Strength \& Conditioning Research, 24, 661-667. http://dx.doi.org/10.1519/JSC.0b013e3181c277a6

[13] Schoffstall, J.E., Titcomb, D.A. and Kilbourne, B.F. (2010) Electromyographic response of the abdominal musculature to varying abdominal exercises. Journal of Strength \& Conditioning Research, 24, 3422-3426. http://dx.doi.org/10.1519/JSC.0b013e3181e74315

[14] Moraes, A.C., Pinto, R.S., Valamatos, M.J., PezaratCorreia, P.L., Okano, A.H., Santos, P.M. and Cabri, J.M. (2009) EMG activation of abdominal muscles in the crunch exercise performed with different external loads. Physical Therapy in Sport, 10, 57-62. http://dx.doi.org/10.1016/j.ptsp.2009.01.001

[15] Aladro-Gonzalvo, A.R., Araya-Vargas, G.A., MachadoDíaz, M. and Salazar-Rojas, W. (2013) Pilates-based exercise for persistent, non-specific low back pain and associated functional disability: A meta-analysis with metaregression. Journal of Bodywork and Movement Therapies, 17, 125-136. http://dx.doi.org/10.1016/j.jbmt.2012.08.003

[16] Beck, R. (2008) Getting to the core. Using Pilates to promote overall wellness during rehabilitation. Rehab Management, 21, 34,36.

[17] Cruz-Ferreira, A., Fernandes, J., Gomes, D., Bernardo, L.M., Kirkcaldy, B.D., Barbosa, T.M. and Silva, A. (2011) Effects of Pilates-based exercise on life satisfaction, physical self-concept and health status in adult women. Women \& Health, 51, 240-255. http://dx.doi.org/10.1080/03630242.2011.563417

[18] Culligan, P.J., Scherer, J., Dyer, K., Priestley, J.L., Guingon-White, G., Delvecchio, D. and Vangeli, M. (2010) A randomized clinical trial comparing pelvic floor muscle training to a Pilates exercise program for improving pelvic muscle strength. International Urogynecology Journal, 21, 401-408. http://dx.doi.org/10.1007/s00192-009-1046-Z

[19] Geweniger, V. (2002) Prevention of back pain with Pilates training: Finding a healthy balance. Pflegezeitschrift, 55, 747-749.

[20] Critchley, D.J., Pierson, Z. and Battersby, G. (2011) Ef- fect of Pilates mat exercises and conventional exercise programmes on transversus abdominis and obliquus internus abdominis activity: Pilot randomised trial. Manual Therapy, 16, 183-189.

http://dx.doi.org/10.1016/j.math.2010.10.007

[21] Loss, J.F., Melo, M.O., Rosa, C.H., Santos, A.B., La Torre, M. and Silva, Y.O. (2010) Electrical activity of external oblique and multifidus muscles during the hip flexion-extension exercise performed in the Cadillac with different adjustments of springs and individual positions. Revista Brasileira de Fisioterapia, 14, 510-517. http://dx.doi.org/10.1590/S1413-35552010000600010

[22] Flint, M.M. (1965) Abdominal muscle involvement during the performance of various forms of sit-up exercise. An electromyographic study. American Journal of Physical Medicine, 44, 224-234.

[23] Floyd, W.F. and Silver, P.H. (1950) Electromyographic study of patterns of activity of the anterior abdominal wall muscles in man. Journal of Anatomy, 84, 132-145.

[24] Reid, G., Montgomery, D.L. and Seidl, C. (1985) Performance of mentally retarded adults on the Canadian Standardized Test of Fitness. Canadian Journal of Public Health, 76, 187-190.

[25] Kim, S.J., Kwon, O.Y., Yi, C.H., Jeon, H.S., Oh, J.S., Cynn, H.S. and Weon, J.H. (2011) Comparison of abdominal muscle activity during a single-legged hold in the hook-lying position on the floor and on a round foam roll. Journal of Athletic Training, 46, 403-408.

[26] Hermens, H.J., Freriks, B., Disselhorst-Klug, C. and Rau, G. (2000) Development of recommendations for SEMG sensors and sensor placement procedures. Journal of Electromyography \& Kinesiology, 10, 361-374. http://dx.doi.org/10.1016/S1050-6411(00)00027-4

[27] Marques, N.R. (2012) EMG activity of trunk stabilizar muscle during centering principle of Pilates method. Journal of Bodywork \& Movement Therapies, 11, 1-7.

[28] Clark, K.M., Holt, L.E. and Sinyard, J. (2003) Electromyographic comparison of the upper and lower rectus abdominis during abdominal exercises. Journal of Strength \& Conditioning Research, 17, 475-483.

[29] Piering, A.W., Janowski, A.P., Wehrenberg, W.B., Moore, M.T. and Snyder, A.C. (1993) Electromyographic analysis of four popular abdominal exercises. Journal of Athletic Training, 28, 120-126.

[30] Hildenbrand, K. and Noble, L. (2004) Abdominal muscle activity while performing trunk-flexion exercises using the Ab Roller, ABslide, FitBall, and conventionally performed trunk curls. Journal of Athletic Training, 39, 37 43.

[31] Lehman, G.J. and McGill, S.M. (2001) Quantification of the differences in electromyographic activity magnitude between the upper and lower portions of the rectus abdominis muscle during selected trunk exercises. Physical Therapy, 81, 1096-1101.

[32] Ozer Kaya, D., Duzgun, I., Baltaci, G., Karacan, S. and Colakoglu, F. (2012) Effects of calisthenics and Pilates exercises on coordination and proprioception in adult women: A randomized controlled trial. Journal of Sport Rehabilitation, 21, 235-243. 
[33] Comfort, P., Pearson, S.J. and Mather, D. (2011) An electromyographical comparison of trunk muscle activity during isometric trunk and dynamic strengthening exercises. Journal of Strength \& Conditioning Research, 25, 149154. http://dx.doi.org/10.1519/JSC.0b013e3181fb412f

[34] Masuda, T., Kizuka, T., Zhe, J.Y., Yamada, H., Saitou, K., Sadoyama, T. and Okada, M. (2001) Influence of contraction force and speed on muscle fiber conduction velocity during dynamic voluntary exercise. Journal of Electromyography \& Kinesiology, 11, 85-94. http://dx.doi.org/10.1016/S1050-6411(00)00048-1

[35] Bloomer, R.J. and Goldfarb, A.H. (2004) Anaerobic exercise and oxidative stress: A review. Canadian Journal of Applied Physiology, 29, 245-263. http://dx.doi.org/10.1139/h04-017

[36] Pandofe, K.D.M., Andrade, M.C.C.D., Meyer, P.F. and Silva, E.M.D.S. (2006) Relationship between abdominal force, abdominal protusion and lobossacral angle in young women. Fisioterapia em Movimento, 19, 99-104.

[37] (2009) Pilates. Strength for your core and more. Mayo
Clinic Women's HealthSource, 13, 7.

[38] Melo, M.O., Gomes, L.E., Silva, Y.O., Bonezi, A. and Loss, J.F. (2011) Assessment of resistance torque and resultant muscular force during Pilates hip extension exercise and its implications to prescription and progression. Revista Brasileira de Fisioterapia, 15, 23-30. http://dx.doi.org/10.1590/S1413-35552011000100006

[39] Menacho, M.O., Obara, K., Conceição, J.S., Chitolina, M.L., Krantz, D.R., da Silva, R.A. and Cardoso, J.R. (2010) Electromyographic effect of mat Pilates exercise on the back muscle activity of healthy adult females. Journal of Manipulative and Physiological Therapeutics, 33, 672-678. http://dx.doi.org/10.1016/j.jmpt.2010.08.012

[40] Wells, C., Kolt, G.S., Marshall, P., Hill, B. and Bialocerkowski, A. (2013) Effectiveness of Pilates exercise in treating people with chronic low back pain: A systematic review of systematic reviews. BMC Medical Research Methodology, 13, 7.

http://dx.doi.org/10.1186/1471-2288-13-7 